\title{
Isolamento do ruído de impacto de pisos em baixa frequência com fontes normalizada e não normalizada
}

\author{
Low frequency impact noise floors insulation by source \\ of impact noise
}

\section{Ana Maria Schuch Araújo Stephan Paul Erasmo Felipe Vergara}

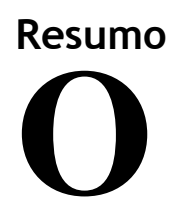
sistema piso flutuante. Foram realizadas medições com duas fontes geradoras de ruído de impacto, sendo uma máquina de impacto normalizada modificada e uma não normalizada (pera de $5 \mathrm{~kg}$ ). Três tipos de piso (laje em osso, cerâmico e laminado) e dois materiais resilientes (lã de vidro e EVA) e quatro posições de fonte de ruído formaram as combinações nas medições realizadas. A partir dos dados analisados, onde foram realizadas análises com 4, 12 e 16 posições de microfone, concluiu-se que para a fonte normalizada o nível de pressão de ruído de impacto $(\mathrm{Ln}, \mathrm{T})$ apresentou variações conforme o tipo de material resiliente utilizado, já para a fonte não normalizada os resultados do nível de pressão sonora de impacto máximo (Li,Fmax) se diferem pelo tipo de piso usado, principalmente na banda de $80 \mathrm{~Hz}$. Neste caso o material resiliente influencia também nos níveis de pressão sonora, mas não tanto quanto influencia nos níveis de pressão sonora da fonte padronizada.

Palavras-chaves: Ruído de impacto em pisos. Baixas frequências. Material resiliente.
Ana Maria Schuch Araújo Universidade Federal da Fronteira Sul Erechim - RS - Brasil

Stephan Paul Universidade Federal de Santa
Catarina Joinville - SC - Brasil

Erasmo Felipe Vergara Universidade Federal de Santa Catarina
Florianópolis - SC - Brasil

Recebido em 18/03/15 Aceito em 07/08/15

\begin{abstract}
The objective of this study was to evaluate the sound pressure level generated by different types of impact noise sources in buildings at frequencies between 80 - 400 $\mathrm{Hz}$ using different floor combinations and resilient materials for the flooring system. Measurements were conducted using two sources of impact noise, a tapping machine modified according to international standards and a nonstandard $5 \mathrm{~kg}$ punching bag. Three types of floor (bare slab, ceramic flooring and laminated flooring) and two resilient materials (glass wool and EVA) were used. Impact noise sources were placed in four different positions. Data were collected at 16 microphone positions in the receiving room, and analyses were performed for 4, 12 and 16 microphone positions. It was concluded that the impact sound pressure level $(L n T)$ present in the receiving room under excitation with the modified tapping machine varies according to the type of resilient material used, while for the non-standard impact noise source the results in terms of maximum impact sound pressure level ( $\mathrm{Li}$, max) vary largely according to the different types of flooring used, especially in the $80 \mathrm{~Hz}$ band. In this case, the resilient material also influences the sound pressure levels, but not as much as it influences the pressure levels of the standard source.
\end{abstract}

Keywords: Noise impact floors. Low frequencies. Resilient material. 


\section{Introdução}

O ruído de impacto e seu isolamento nas edificações, principalmente nas residenciais, têm sido alvo de muitos estudos, entretanto as pesquisas não têm alcançado êxito quanto ao incômodo causado por esse tipo de ruído. O uso crescente de aparelhos capazes de gerar ruído com mais energia em baixas frequências, abaixo de 200 $\mathrm{Hz}$, tais como os sistemas de áudio com alta potência e resposta aprimorada das frequências graves, serviços mecânicos, dispositivos elétricos, e construções cada vez mais leves, tem ocasionado o agravamento do ruído de impacto.

Embora existam muitos esforços para reduzir o ruído de impacto nos pisos das construções, ainda não foi definido um método eficiente que seja capaz de isolar o ruído de impacto causado nas baixas frequências, isto é, de 20 a $200 \mathrm{~Hz}$. Um dos aspectos que contribuem para isso é o fato da própria máquina de impactos padronizada ser deficiente para excitar essa faixa de frequência (SHI; JOHANSSON; SUNDBÄCK, 1997; WARNOCK, 1998, 1992, 2000; JEON, 2001; JEON et al., 2004; BRADLEY, 2004; JEON; SATO, 2008; KIM et al., 2009; YOO et al., 2010; SCHOENWALD et al., 2010; SCHOENWALD; ZEITLER; NIGHTINGALE, 2010; NEVES E SOUSA; GIBBS, 2011; CABEÇAS, 2011). Outro fator a ser ressaltado é que as frequências centrais, em banda de terço de oitava para análise, estabelecidas nas normas ISO (INTERNATIONAL..., 2010a， 2010b， 2010c, 2010d; SHI; JOHANSSON; SUNDBÄCK, 1997), são superiores a $100 \mathrm{~Hz}$ e sabe-se que a audição humana consegue detectar sons entre 20 e 20.000 $\mathrm{Hz}$.

Desde 2010, as normas ISO 10140-3 (INTERNATIONAL..., 2010b) e ISO 10140-4 (INTERNATIONAL..., 2010c) incluem a possibilidade de medir a partir de $50 \mathrm{~Hz}$, quando forem necessárias informações adicionais nas faixas de baixa frequência. Embora a medição não seja obrigatória, verifica-se a necessidade de seu estudo ser obrigatório em normas e regulamentos.

Este artigo tem por finalidade investigar o comportamento das combinações de pisos e materiais resilientes quando excitados por uma fonte padronizada (máquina de impactos modificada) e uma fonte de impacto não padronizada (pera de $5 \mathrm{~kg}$ ). Na literatura os autores apenas se referem a sacos ou bolas de areia, mas não a qual material é usado na composição do saco. A escolha da pera de $5 \mathrm{~kg}$ se deu em função do peso. Tentou-se fazer medições com um saco de areia de $3 \mathrm{~kg}$, todavia o saco estourou na terceira medição. Foram realizadas avaliações experimentais do isolamento do ruído de impacto de acordo com as normas ISO 10140-3:2010 e 10140-5:2010, dando enfoque para a faixa de frequências centrais entre 80 e $400 \mathrm{~Hz}$. Esse procedimento foi adotado para aprimorar a avaliação do desempenho do isolamento, já que a máquina de impacto normalizada não reproduz com fidelidade o caminhar de pessoas e fontes de impacto de baixa frequência.

\section{Método}

A pesquisa foi desenvolvida no Laboratório de Acústica da Universidade Federal de Santa Maria (UFSM) e foram utilizadas dois tipos de fonte de ruído de impacto, a primeira uma máquina de impacto modificada (MIM) ${ }^{1}$ (Figura 1) do tipo leve. A fonte MIM caracteriza-se por ser uma fonte dinâmica simulando uma pessoa caminhando descalça e uma fonte de impacto pesado/leve com características de fonte dinâmica semelhante à de crianças pulando. O Anexo F da ISO 10140-5 (INTERNATIONAL..., 2010d) especifica dois métodos para a criação de uma máquina de impacto modificada; no Método A as molas são fixadas aos martelos da máquina de impacto padrão; e no Método B coloca-se uma camada macia no piso sob a área do martelo da máquina de impacto. A segunda fonte de impacto utilizada foi do tipo saco em formato de pera de $5 \mathrm{~kg}^{2}$ (Figura 1), considerada fonte de impacto pesado. Geralmente, os sacos de areia são bons geradores de ruído sonoro de impacto em baixas frequências, conseguindo produzir ruídos de impacto com espectros de frequência de pessoas caminhando (SHI; JOHANSSON; SUNDBÄCK, 1997).

\footnotetext{
${ }^{1} \mathrm{~A}$ MIM utiliza quatro camadas de feltro, de $45 \mathrm{~cm} \times 5 \mathrm{~cm}$, entre os martelos da máquina e o piso. 0 feltro usado foi o Feltycril, da Feltros Santa Fé, com $1 \mathrm{~mm}$ de espessura e $0,185 \mathrm{~g} / \mathrm{cm}^{3} \mathrm{de}$ densidade.

${ }^{2}$ Pera para prática de boxe da marca Knockout, linha profissional, código 402, confeccionada em couro natural de alta resistência, forrada em lona e com enchimento de resíduo de pano e peso de $5 \mathrm{~kg}$. Quando a pera bate no chão ela quica e sobe no ar uns $15 \mathrm{~cm}$, para cair novamente no chão e permanecer parada.
} 
Figura 1 - Fontes sonoras geradoras de ruído de impacto

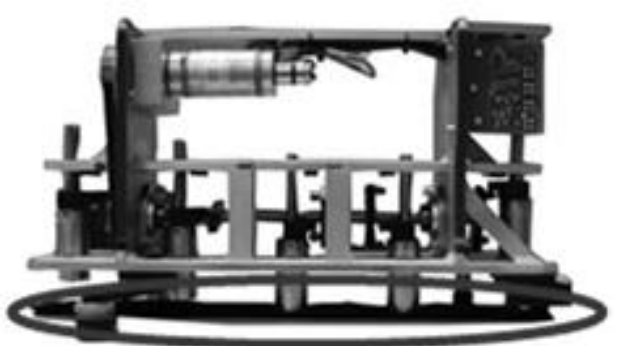

(a) Máquinas de impacto modificada (MIM)

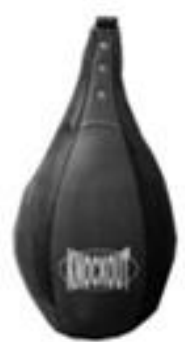

(b) Pera de $5 \mathrm{~kg}$
Cada fonte de ruído de impacto excitou diferentes tipos de combinação laje-piso: 1) laje em osso; 2) laje mais contrapiso de concreto com $3,5 \mathrm{~cm}$ de espessura; 3) laje mais contrapiso de concreto com $3,5 \mathrm{~cm}$ de espessura com piso cerâmico; e 4) laje mais contrapiso de concreto com $3,5 \mathrm{~cm}$ de espessura com piso laminado. Os contrapisos utilizados possuem área de $1 \mathrm{~m}^{2}$. Além disso, foram utilizados os seguintes materiais resilientes, uma amostra de lã de vidro ${ }^{3}$ e uma de EVA $^{4}$, ambos bastante utilizados na construção civil brasileira, inclusive nas espessuras aqui ensaiadas.

As medições foram consideradas in situ, uma vez que as salas do laboratório para a realização desse experimento não atendem a todos os critérios da norma ISO 140-6. Como a fonte pera não é normalizada, os experimentos foram realizados de acordo com os métodos da norma ISO 10140 5:2010 e recomendações de estudos sobre ruído de impacto em pisos e fontes tipo bola (SHI; JOHANSSON; SUNDBÄCK, 1997; WARNOCK, 1998, 1992, 2000; JEON, 2001; JEON et al., 2004; BRADLEY, 2004; JEON; SATO, 2008; KIM et al., 2009; YOO et al., 2010; SCHOENWALD et al., 2010). As medições com a pera foram efetuadas utilizando-se um mecanismo que permitiu segurá-la e soltá-la a $1 \mathrm{~m}$ do piso, conforme recomenda o Anexo F da ISO 10140-5 (INTERNATIONAL..., 2010d).

As fontes geradoras de impacto foram posicionadas conforme exigência da ISO 10140-3 e ISO 10140-5 (INTERNATIONAL..., 2010b, 2010d) na sala emissora (Figura 2). O tempo médio para a obtenção dos níveis de pressão sonora de impacto foi de $15 \mathrm{~s}$ para cada posição de microfone.
Segundo as exigências da norma ISO 10140-3 (INTERNATIONAL..., 2010b), seriam possíveis 15 posições de microfone na sala receptora, porém o número de microfones deve ser igual ou múltiplo inteiro do número de posições de fonte (Figura 3a). Assim, foram efetuados ensaios com quatro posições de fonte geradora de impacto, doze pontos de microfone e duas repetições de medição para cada combinação de fonte/receptor, conforme a Norma ISO 10140-4 (INTERNATIONAL..., 2010c), nas nove combinações de piso/material resiliente, totalizando 864 medições para cada tipo de fonte geradora de ruído de impacto.

Os ensaios experimentais foram efetuados segundo o Anexo A da ISO 10140-4 (INTERNATIONAL..., 2010c) e observando os procedimentos adicionais para medições em baixas frequências (Figura 3b). As dimensões da câmara receptora comportam seis pontos de microfone, mas novamente o número de posições de microfone deve ser igual ou múltiplo inteiro do número de posições de fonte geradora de ruído de impacto. Dessa forma, foram realizadas medições adicionais com quatro posições de fonte geradora de impacto, quatro pontos de microfone e duas repetições de medição para cada combinação de fonte/receptor para as nove combinações de piso/material resiliente, totalizando assim 288 medições adicionais de nível de pressão sonora de impacto para cada tipo de fonte geradora de ruído de impacto. No total foram realizadas 2.304 medições de nível de pressão sonora.

Para a fonte MIM determinou-se o nível de pressão sonora de impacto normalizado $\left(L_{\mathrm{n}, \mathrm{T}}\right)$, e para quantificar o ruído gerado pela pera foi utilizado o nível de pressão sonora máximo $\left(L_{\mathrm{i}, \mathrm{Fmax}}\right)$ medido com tempo de integração Fast (Figura 4).

\footnotetext{
${ }^{3}$ Amostra de lã de vidro com 1,10 m x 1,10 m de área da Wallfelt 50 , recomendada para o preenchimento de paredes drywall da Isover Saint Gobain, com $50 \mathrm{~mm}$ de espessura e $10 \mathrm{~kg} / \mathrm{m}^{3} \mathrm{de}$ densidade.

${ }^{4}$ Amostra de EVA 1826 (preto) com 1,00 m x 1,50 m de área da Tacosola Borrachas Ltda., com $10 \mathrm{~mm}$ de espessura, $0,18 \mathrm{~kg} / \mathrm{m}^{3}$ de densidade e $\mathbf{2 6}$ de dureza Shore A.
} 
Figura 2 - Posições de fonte geradora de ruído de impacto

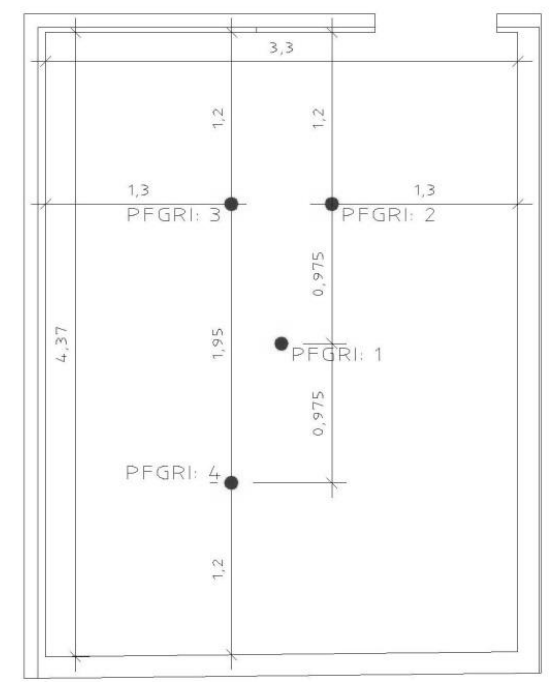

Figura 3 - Posições de microfone: (a) segundo a ISO 10140-3:2010 e (b) segundo o Anexo A da ISO 10140-4:2010

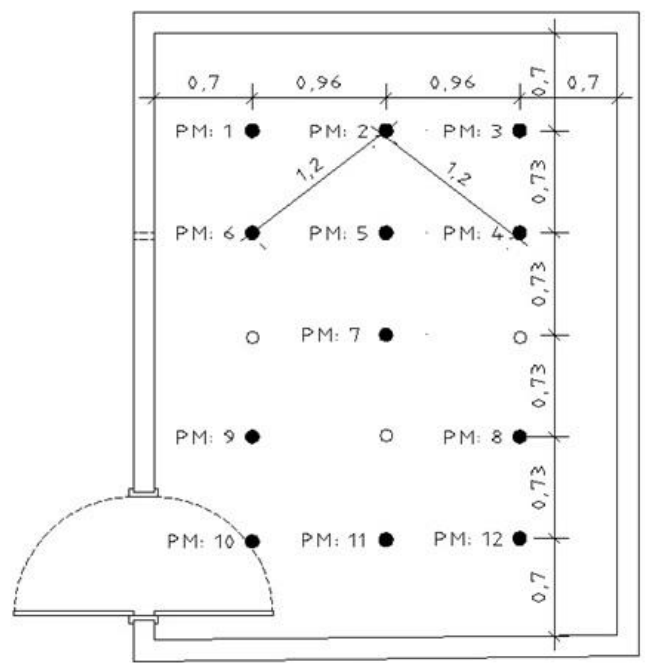

a)

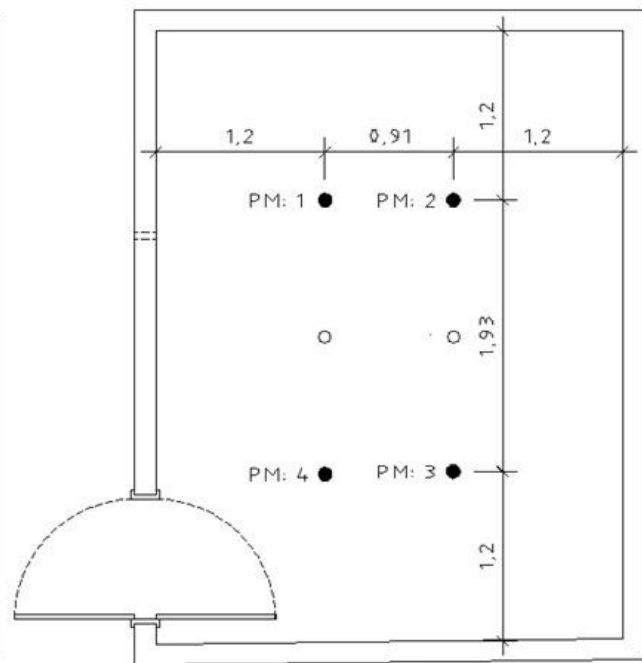

b)

Nota: Legenda:

posições de microfone utilizadas; e

- posições de microfone descartadas.

Figura 4 - Nível de pressão sonora equivalente $\left(L_{\text {Aeq }}\right)$ no tempo da pera na combinação pera + laje em osso na posição de fonte 1 e posição de microfone 3 (12 pontos de microfone)

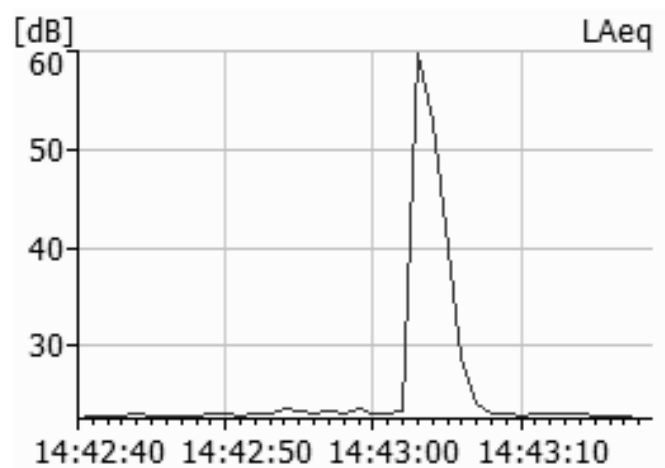

166 Araújo, A. M. S.; Paul. S.; Vergara, E. F. 
Todavia, para a execução das medições foram utilizados os seguintes equipamentos:

(a) máquina de impacto Brüel \& Kjaer do tipo 3207;

(b) amplificador de potência Brüel \& Kjaer do tipo 2716; fonte sonora omnidirecional Brüel \& Kjaer do tipo 4292, para emissão de ruído rosa de banda larga;

(c) medidor de temperatura, CE, NF: 171394-R;

(d) medidores do nível de pressão sonora Brüel \& Kjaer do tipo 2270; e

(e) microfones Brüel \& Kjaer, de meia polegada do tipo 4189, com equalização para campo difuso.

Para registrar o nível de pressão sonora do ruído de fundo foi utilizado o medidor do nível de pressão sonora com o ambiente sem equipamentos geradores de ruído. O microfone foi posicionado a uma altura de $1,30 \mathrm{~m}$ do chão. $\mathrm{O}$ ruído de fundo sempre foi medido após as duas medições de nível de pressão sonora do ruído de impacto em cada combinação microfone-fonte-piso-material resiliente. Além disso, em função de a atenuação sonora na propagação sonora ser inversamente proporcional à temperatura e umidade, os experimentos foram feitos nas condições de temperatura e umidade exigidas pelas normas: entre 18 e $25^{\circ} \mathrm{C}$ e com umidade relativa inferior a $80 \%$. Os valores médios de temperatura permaneceram entre 18 e $20^{\circ} \mathrm{C}$, e os da umidade relativa do ar variaram entre $65 \%$ e $72 \%$.

\section{Resultados}

A análise dos dados obtidos nas medições foi realizada em duas etapas: a primeira com os dados obtidos com a fonte padronizada MIM; e a segunda com os dados da fonte não normalizada, a pera de $5 \mathrm{~kg}$. Os resultados são apresentados separadamente para as medições com as combinações com contrapiso de concreto, com piso cerâmico, com piso laminado e a comparação entre 12,4 e 16 pontos de microfone.

\section{Fonte padronizada MIM}

Ao analisar os níveis de pressão sonora de impacto normalizado $\left(L_{\mathrm{n}, \mathrm{T}}\right)$ produzidos pela fonte MIM (Figuras 5, 6, e 7) observa-se, primeiramente, que as combinações de pisos com lã de vidro (LV) se diferem das outras combinações que usam EVA e das que não possuem material resiliente, sendo que a lã de vidro proporcionou maior isolamento ao ruído de impacto independentemente do tipo de piso. A espessura, como característica do material, pode estar determinando esse comportamento, pois, enquanto a lã de vidro tem $5 \mathrm{~cm}$ de espessura, o EVA possui $1 \mathrm{~cm}$. No entanto, optou-se por esta espessura de EVA por ser a mais vendida no comércio para isolamento acústico de ruído de impacto em pisos.

Observando-se a Figura 5, os gráficos das medições feitas com contrapiso de concreto com 12,4 e 16 posições de microfone (PM), verifica-se que os espectros obtidos com 12 e 16 pontos apresentam o mesmo comportamento, enquanto com quatro posições de microfone há uma pequena diferenciação. Isto é, na banda de $80 \mathrm{~Hz}$ a variação do $\mathrm{L}_{\mathrm{n}, \mathrm{T}}$ oscila em torno de $5 \mathrm{~dB}$; para as bandas de 100 e $200 \mathrm{~Hz}$ permanece ao redor dos $2 \mathrm{~dB}$; e nas demais frequências em torno de $1 \mathrm{~dB}$.

Na Figura 6 pode ser observado que, para as bandas de frequências de 100 e $125 \mathrm{~Hz}$, o piso cerâmico apresentou melhor desempenho no isolamento de ruído de impacto (menor índice $L_{\mathrm{n}, \mathrm{T}}$ ) quando comparado aos outros pisos experimentados, enquanto o piso laminado potencializa a transmissão do ruído de impacto nas mesmas bandas quando comparado com a laje em osso. Entretanto, na banda de $80 \mathrm{~Hz}$ o piso laminado tem um ganho em comparação ao piso cerâmico em torno de $3 \mathrm{~dB}$ em seu desempenho de isolamento de ruído. Em contrapartida, o piso cerâmico nessa mesma banda transmite mais ruído de impacto, variando em torno de $9 \mathrm{~dB}$ para a fonte MIM (Figuras 5, 6, e 7).

Para as medições com piso cerâmico, o comportamento dos espectros não apresenta alterações significativas para 12 e 16 posições de microfone (Figura 6). Pode-se observar que os níveis de pressão sonora de ruído de impacto $\left(L_{\mathrm{n}, \mathrm{T}}\right)$, na faixa entre 80 e $100 \mathrm{~Hz}$, apresentam valores menores quando medidos com quatro posições de microfone (Figura 6c). Entretanto, para 12 e 16 posições de microfone, essas diferenças não são significativas, conforme o teste T pareado; $t$ varia 3,70 .

Para o piso laminado o comportamento dos espectros de frequências centrais não apresenta alterações significativas para 12 e 16 posições de microfone (Figura 7). Pode-se observar que os níveis de pressão sonora de ruído de impacto, da faixa entre 80 e $100 \mathrm{~Hz}$, apresentam valores menores quando medidos com apenas quatro microfones. Entretanto, para 12 e 16 posições de microfone, essas diferenças não são significativas, de acordo com o teste T pareado; $\mathrm{t}$ varia $-0,18$. 
Figura 5 - Níveis de pressão sonora do ruído de impacto $\left(L_{\mathrm{n}, \mathrm{T}}\right)$ da fonte MIM e contrapiso de concreto nas posições de microfone (a) 12, (b) 16 e (c) 4

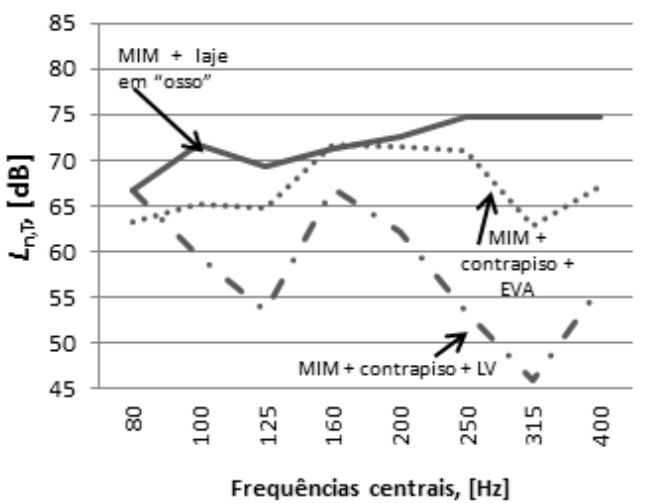

a)

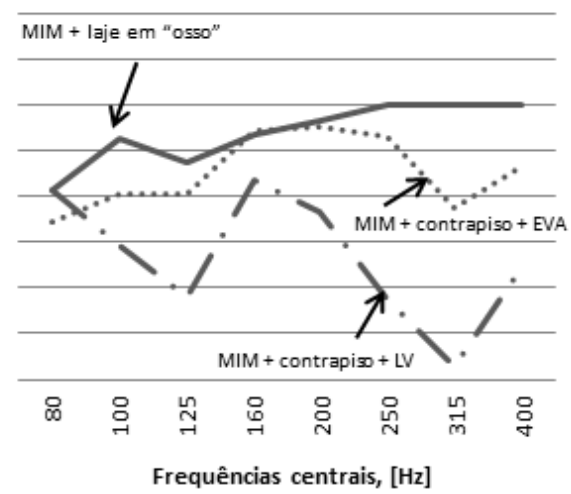

b)

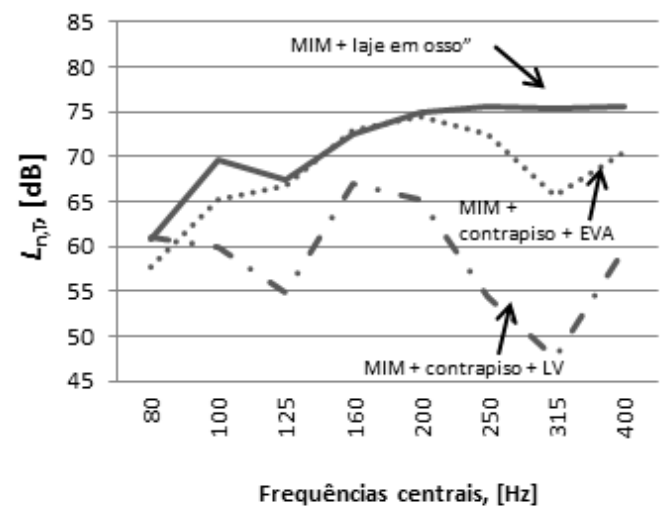

c)

Figura 6 - Níveis de pressão sonora do ruído de impacto $\left(L_{n, T}\right)$ da fonte MIM e piso cerâmico nas posições de microfone (a) 12 , (b) 16 e (c) 4
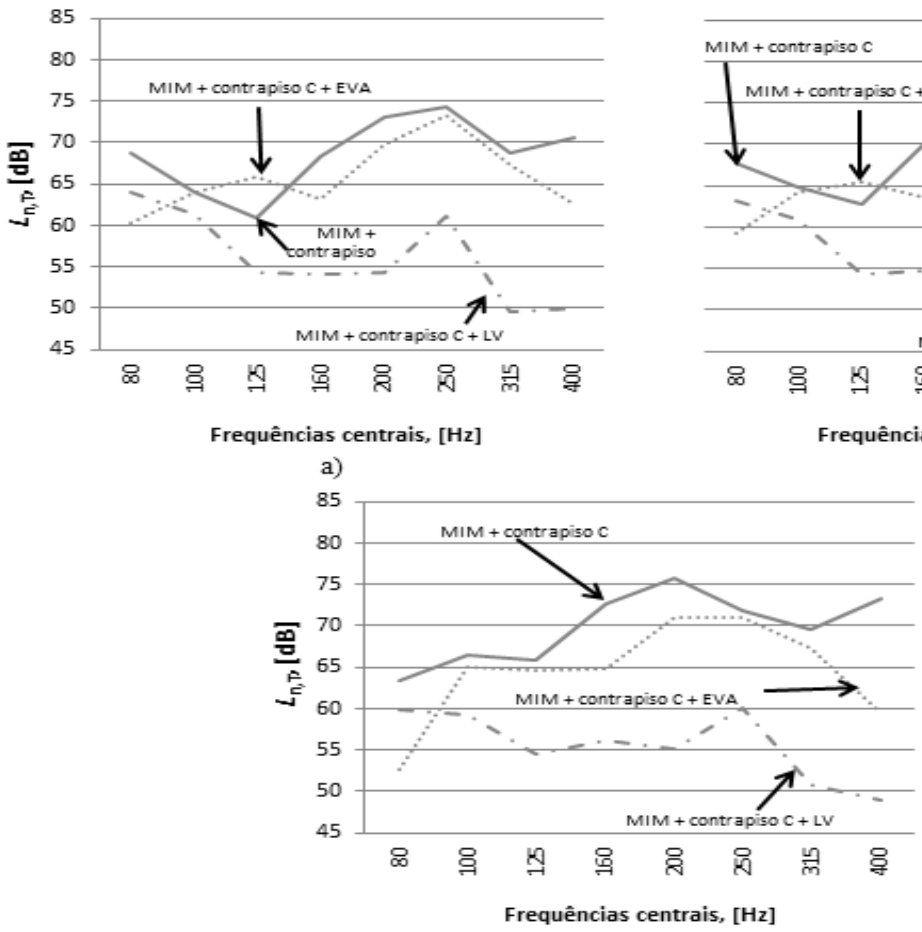

c)

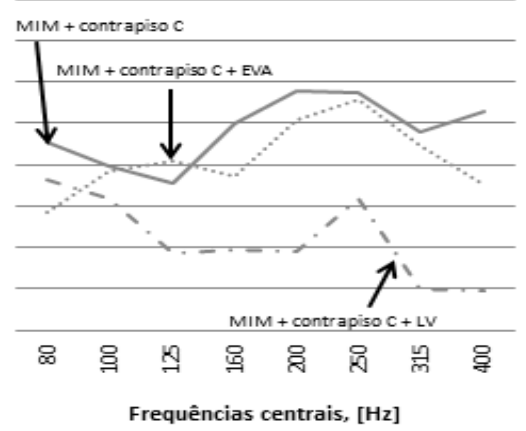

b) 
Figura 7 - Níveis de pressão sonora do ruído de impacto $\left(L_{n, T}\right)$ da fonte MIM e piso laminado nas posições de microfone (a) 12, (b) 16 e (c) 4

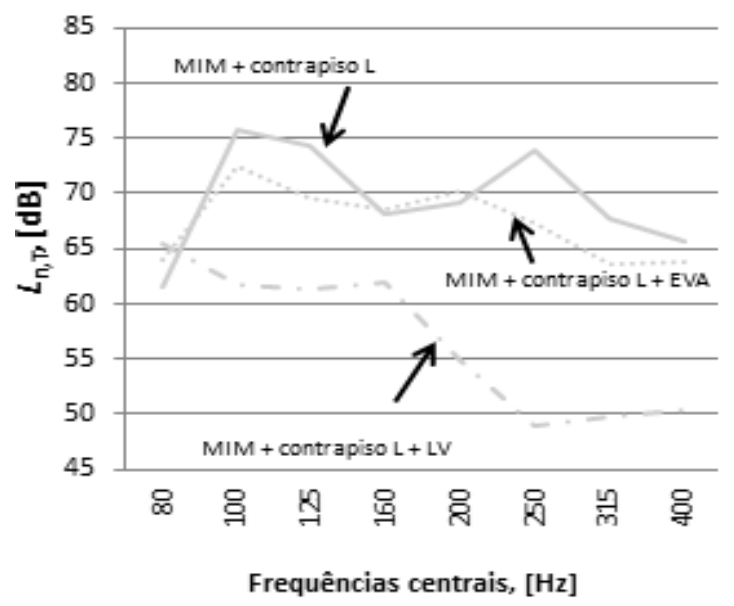

a)

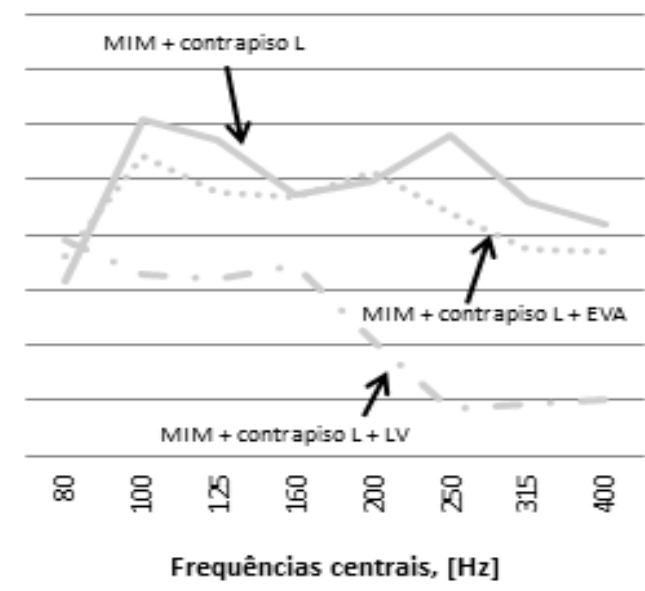

b)

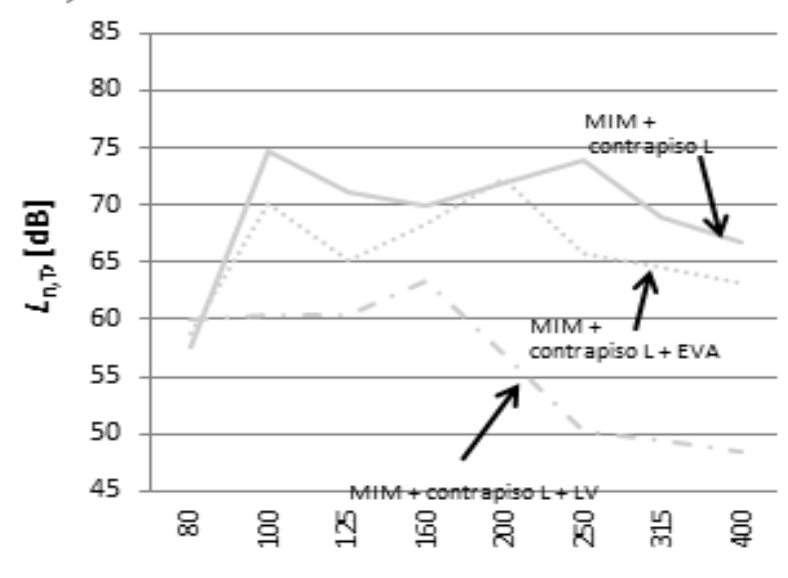

Frequências centrais, $[\mathrm{Hz}]$

c)

Ao analisar os resultados do $\mathrm{L}_{\mathrm{n}, \mathrm{T}}$ obtidos com a fonte MIM para 4 e 12 posições, a maior discrepância está em $80 \mathrm{~Hz}$, uma vez que as diferenças variam entre 4,3 e 7,6 dB. Por outro lado, para as combinações analisadas em outras frequências centrais, a diferença de níveis de pressão sonora varia em torno de $1 \mathrm{~dB}$, com exceção de $200 \mathrm{~Hz}$, que, para a maioria das combinações, o NPS varia em torno de 2,5 dB.

Já as diferenças de níveis de pressão sonora de ruído de impacto para 12 e 16 posições de microfone, em $80 \mathrm{~Hz}$, na maioria dos casos variam em torno de $1 \mathrm{~dB}$ (Figuras 8a e 8c). Além disso, as demais frequências centrais também variam em torno de $1 \mathrm{~dB}$ (Figuras 8a e 8c). No entanto, para a combinação contrapiso cerâmico, há discrepância em $125 \mathrm{~Hz}$, que varia próximo dos $2 \mathrm{~dB}$ (Figura $8 b)$.

Dessa forma, salienta-se que, para os resultados obtidos neste trabalho, o procedimento do Anexo A da ISO 10140-4:2010 pode ser dispensado, visto que as diferenças de valores de nível de pressão sonora de ruído de impacto $\left(\mathrm{L}_{\mathrm{n}, \mathrm{T}}\right)$ são muito pequenas. Como o procedimento do Anexo A da ISO 10140-4 (INTERNATIONAL..., 2010c) é adicional, não se pode comparar separadamente 12 e 4 posições de microfone, e sim confrontar as posições 12 e 16 (os valores somados $12+4=16$ ).

\section{Fonte não padronizada - Pera de 5 kg}

Os níveis de pressão sonora de impacto máximo $\left(L_{\mathrm{i}, \text { Fmax }}\right)$ medidos com tempo de integração Fast para a fonte não padronizada (pera de $5 \mathrm{~kg}$ ) são mostrados nas Figuras 9, 10 e 11. Em geral, pode ser observado que as combinações de pisos sem material resiliente se diferem das combinações com material resiliente, independentemente do tipo de piso usado. É claro notar que as transmissões sonoras nos pisos cerâmico e laminado, sem material resiliente, são maiores do que na laje em osso (Figuras 10 e 11). 
Os resultados da literatura mostram que, com o aumento da espessura da laje (usando o mesmo material), há diminuição do nível de pressão sonora na sala receptora devido ao ruído de impacto produzido na laje. No caso avaliado temos o contrapiso laminado ou cerâmico, com dimensões de $1 \mathrm{~m}$ x $1 \mathrm{~m}$ e menores que a laje $(3 \mathrm{~m}$ x $4 \mathrm{~m}$ ), que foi instalado sobre a laje de concreto A resposta vibratória do contrapiso laminado ou cerâmico, que é diferente da laje, e constitui a excitação da laje. Assim, não há redução da excitação da laje pela presença do contrapiso laminado ou cerâmico.

Figura 8 - Níveis de pressão sonora de impacto normalizado $\left(L_{n, T}\right)$ com a fonte MIM e para 4, 12 e 16 posições de microfone

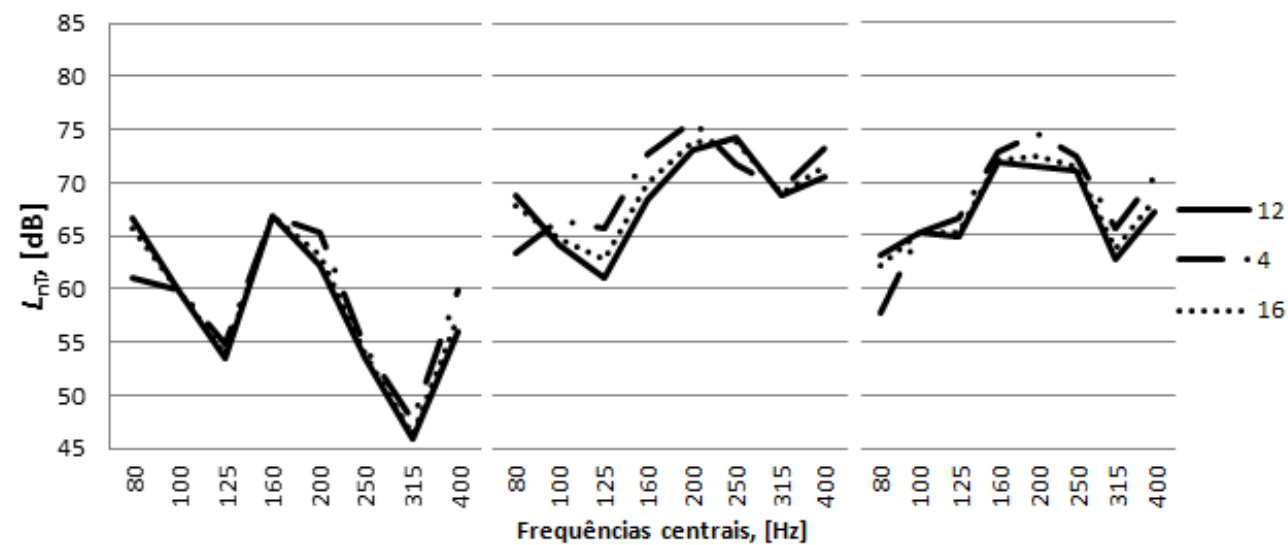

$\begin{array}{lll}\text { (a) Contrapiso+lã de vifro } & \text { (b) Contrapiso cerâmico } & \text { (c) Contrapiso_EVA }\end{array}$

Figura 9 - Nível de pressão sonora de impacto máximo $\left(L_{\mathrm{i}, \mathrm{Fmax})}\right.$ com a fonte pera de $5 \mathrm{~kg}$ e contrapiso de concreto nas posições de microfones (a) 12 (b) 16 e (c) 4

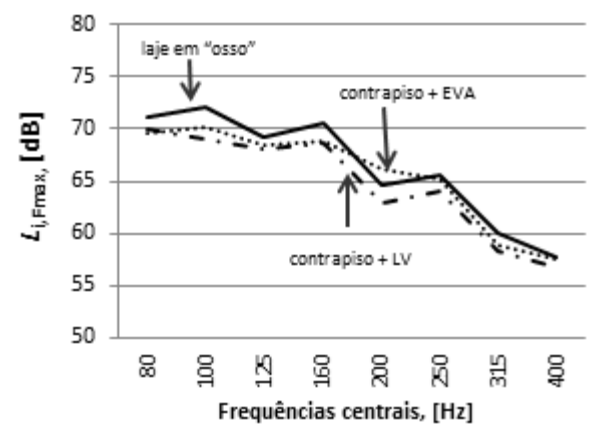

a)

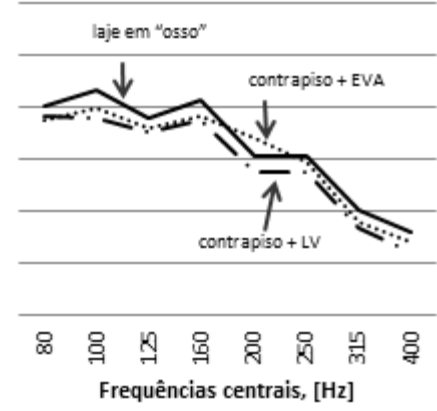

b)

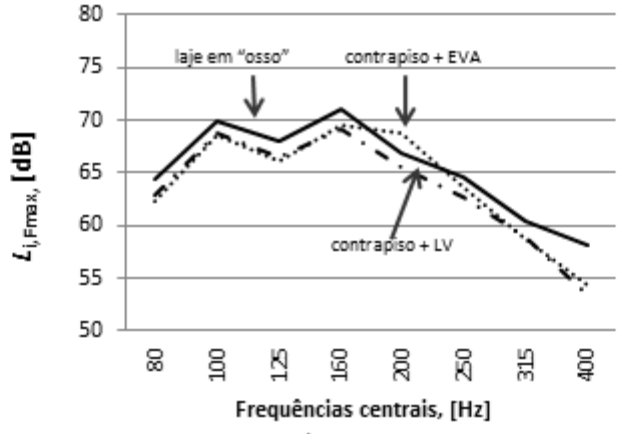

c) 
Figura 10 - Nível de pressão sonora de impacto máximo $\left(L_{\mathrm{i}, \mathrm{Fmax}}\right)$ com a fonte pera de $5 \mathrm{~kg}$ e contrapiso cerâmico (C) nas posições de microfones (a) 12 (b) 16 e (c) 4

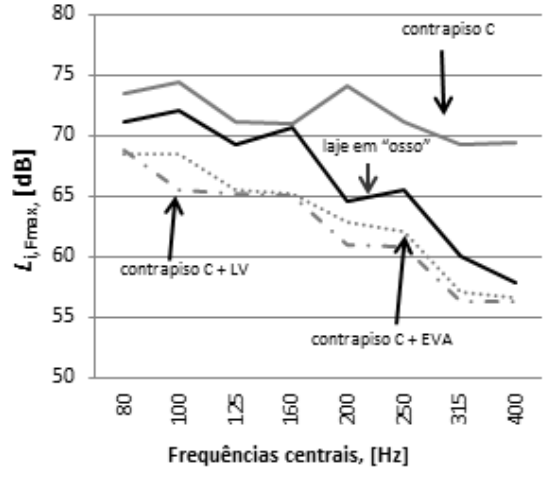

a)

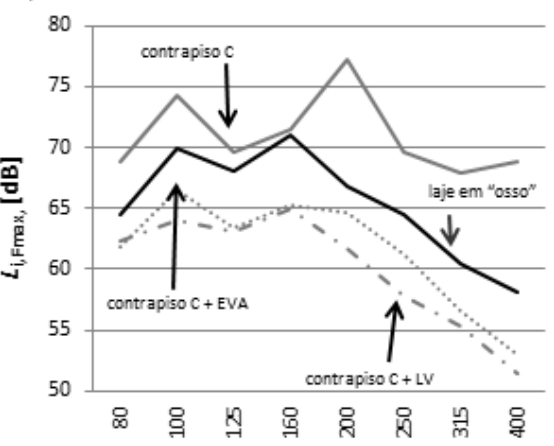

Frequências centrais, $[\mathrm{Hz}]$

c)

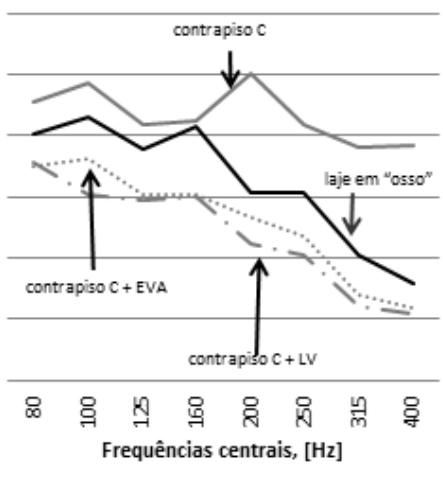

b)

Figura 11 - Nível de pressão sonora de impacto máximo $\left(L_{\mathrm{i}, \mathrm{Fmax}}\right)$ com a fonte pera de $5 \mathrm{~kg}$ e contrapiso laminado (L) nas posições de microfones (a) 12 (b) 16 e (c) 4

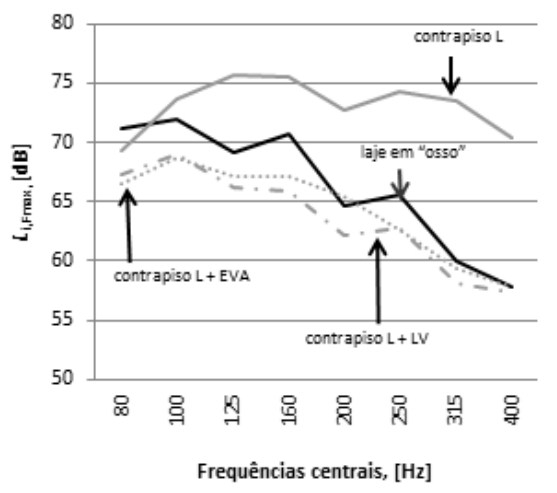

a)

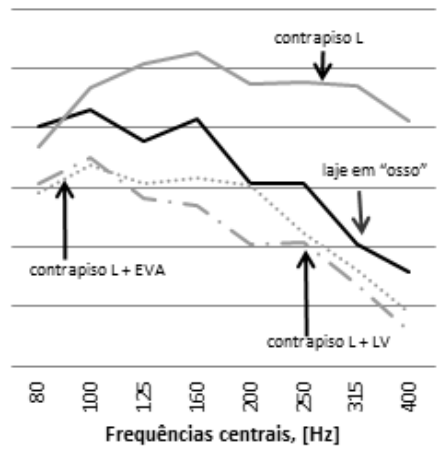

b)

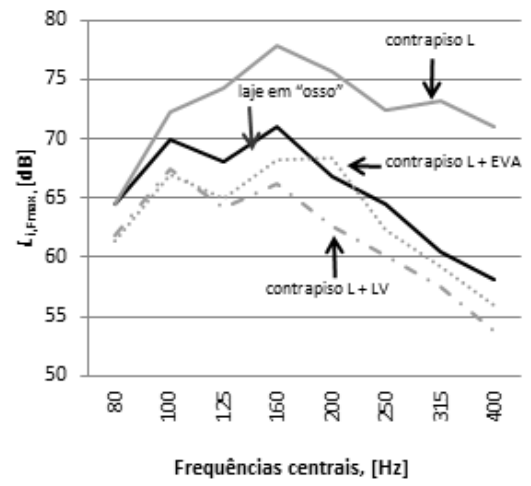

c) 
Além disso, os resultados de medições da impedância mecânica em pisos (SCHOLL 2001; WARNOCK, 1983) mostram que os pisos com e sem contrapisos modificam sua impedância mecânica (relação entre a força aplicada e a resposta de velocidade do sistema: laje ou laje + contrapiso) e varia com a frequência, dependendo da massa e da rigidez do sistema (laje + contrapiso) e da frequência de ressonância do sistema pera + contrapiso.

Quando os materiais resilientes, lã de vidro e EVA, são incorporados aos pisos cerâmico e laminado, os níveis de pressão sonora se reduzem e se aproximam daqueles obtidos com a laje em osso. Esses resultados indicam que as características desses materiais resilientes produzem um ganho no isolamento ao ruído de impacto na maioria das bandas de frequência entre 80 e $400 \mathrm{~Hz}$, com exceção das bandas de 160 e $315 \mathrm{~Hz}$ para o contrapiso e EVA e na banda de $200 \mathrm{~Hz}$ para o contrapiso laminado e EVA. Comparativamente, a lã de vidro em relação ao EVA foi o material resiliente que proporcionou melhor isolamento acústico independentemente do tipo de piso utilizado.

Para as combinações do contrapiso de concreto com lã de vidro e EVA se verifica na Figura 9 que os espectros de frequências do $L_{\mathrm{i}, \mathrm{Fmax}}$, para as posições de microfone 12 e 16, variam em torno de $1 \mathrm{~dB}$. Para quatro posições de microfones as maiores variações do $L_{\mathrm{i}, \mathrm{Fmax}}$ se apresentam na banda de $80 \mathrm{~Hz}$, entre 6 e 7,5 dB, e na banda de $400 \mathrm{~Hz}$, entre 2 e $3 \mathrm{~dB}$.

As combinações com piso cerâmico e a aplicação da lã de vidro e do EVA provocaram redução dos $L_{\mathrm{i}, \text { Fmax }}$ para as três posições de microfones utilizadas (Figura 10). Para as combinações com 12 e 16 posições os níveis de pressão sonora decaíram de 4 a $6 \mathrm{~dB}$, na faixa entre 80 e $160 \mathrm{~Hz}$, e de 9 a $14 \mathrm{~dB}$ entre 200 e $400 \mathrm{~Hz}$, enquanto para quatro posições a diminuição do $L_{\mathrm{i}, \mathrm{Fmax}}$ foi em torno de 6 para 80, 125 e $160 \mathrm{~Hz}, 7 \mathrm{~dB}$ para a combinação piso cerâmico e a aplicação do EVA, e $10 \mathrm{~dB}$ para a combinação piso cerâmico e a aplicação da lã de vidro, ambos em $100 \mathrm{~Hz}$, e de 8 a $17 \mathrm{~dB}$ na faixa entre 200 e $400 \mathrm{~Hz}$.

Para o piso laminado com lã de vidro e EVA também é observada na Figura 11 uma tendência na diminuição dos $L_{\mathrm{i}, \mathrm{Fmax}}$, em torno de 2 a $6 \mathrm{~dB}$ para 80 e $100 \mathrm{~Hz}$, de 7 a $17 \mathrm{~dB}$ na faixa de 125 a $400 \mathrm{~Hz}$, para 12 e 16 posições de microfone. Quando as posições de microfone são 4, os níveis de pressão sonora decaíram em torno de $3 \mathrm{~dB}$ para $80 \mathrm{~Hz}$, de 4 a $17 \mathrm{~dB}$ para a faixa entre 100 e 400 $\mathrm{Hz}$.

Ao comparar o $\mathrm{L}_{\mathrm{i}, \mathrm{Fmax}}$ em função do número das posições de microfones para os contrapisos cerâmico, laminado com lã de vidro e laminado com EVA, é observado na Figura 12 que as maiores diferenças alcançaram 3 a $6 \mathrm{~dB}$ nas bandas de 80 e $200 \mathrm{~Hz}$, e para as outras bandas a diferença predominou em 1,5 dB. Os níveis de pressão sonora para o contrapiso cerâmico variaram entre 67 e 75,6 dB e alcançaram os maiores valores em 100 e 200 Hz, porém a maior variação de NPS está em $80 \mathrm{~Hz}$. Para o contrapiso laminado com lã de vidro o $L_{\mathrm{i}, \mathrm{Fmax}}$ diminui de 3 a $5 \mathrm{~dB}$ em de $80 \mathrm{~Hz}$, e para o contrapiso laminado com EVA o $L_{\mathrm{i}, \mathrm{Fmax}}$ decresce de 3 a $5 \mathrm{~dB}$ em $80 \mathrm{~Hz}$; ficou entre 60 e $68,6 \mathrm{~dB}$ na faixa entre 80 e $250 \mathrm{~Hz}$; e permaneceu abaixo dos $60 \mathrm{~dB}$ em 315 a $400 \mathrm{~Hz}$.

Figura 12 - Nível de pressão sonora de impacto máximo $\left(L_{i, F m a x)}\right.$ com a fonte pera de 5 kg nas posições de medição 12,4 e 16 para os contrapisos

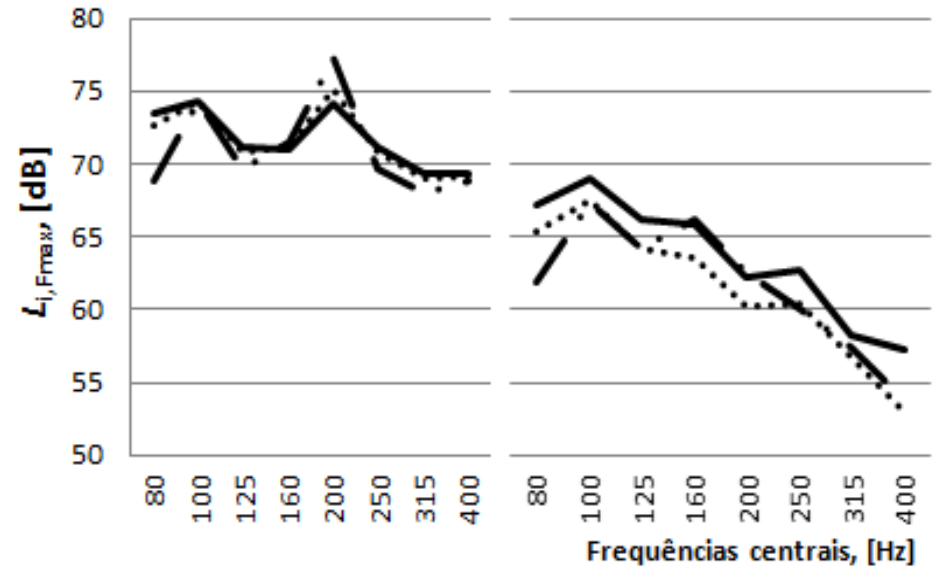

(a) Cerâmico (b) Laminado+lã de vidro

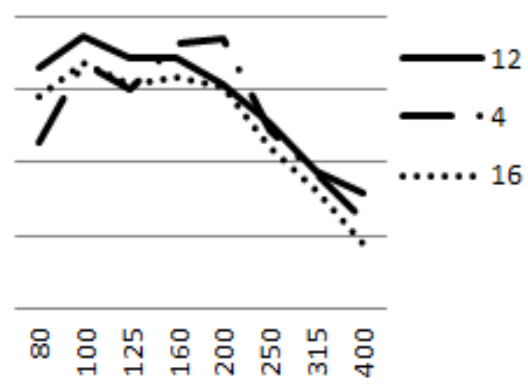

(c) Laminado+EVA

172 Araújo, A. M. S.; Paul. S.; Vergara, E. F. 


\section{Conclusões}

A partir deste estudo constatou-se que os procedimentos para avaliação do ruído de impacto em pisos devem abranger frequências inferiores a $100 \mathrm{~Hz}$, para que as baixas frequências sejam compreendidas integralmente, uma vez que a audição humana consegue detectar sons entre no mínimo $20 \mathrm{~Hz}$ e $20.000 \mathrm{~Hz}$. No entanto, o sistema auditivo humano é pouco sensível aos sons de baixas frequências. Basta lembrar as curvas de audibilidade e a curva de ponderação A. Para se chegar à conclusão de que os procedimentos devem abranger frequências inferiores a $100 \mathrm{~Hz}$, deveriam ser apresentados os níveis de ruído de impacto para as frequências acima de $100 \mathrm{~Hz}$ e então verificar se faz mesmo diferença ter ou não as componentes abaixo de $100 \mathrm{~Hz}$. Além disso, verificou-se a necessidade de se estipular ao menos uma fonte geradora de ruído de impacto pesado, como, por exemplo, a fonte pera de $5 \mathrm{~kg}$, já que ela simula melhor o caminhar das pessoas, enquanto a máquina de impacto normalizada é uma fonte geradora de impacto leve e não representa fielmente impactos pesados.

Foi verificado que para a máquina de impacto normalizada modificada a diferença entre os níveis de pressão sonora não varia para os diferentes contrapisos avaliados: contrapiso de concreto, contrapiso cerâmico e contrapiso laminado, mas varia em função do tipo de material resiliente usado. Para a fonte pera de $5 \mathrm{~kg}$ o tipo de contrapiso influenciou na transmissão do ruído de impacto devido ao baixo amortecimento e à maior rigidez dos contrapisos, cerâmico e laminado, sem a aplicação do material resiliente.

Os sistemas laje + contrapiso (concreto denso) possuem baixo fator de amortecimento interno para as ondas de flexão - implica maior radiação sonora do ruído de impacto em pisos, com valor do fator de amortecimento em torno de 0,005 para o concreto (HOPKINS, 2007) -, porém possuem alta rigidez e menor impedância mecânica, o que favorece a transmissão de ruído de impacto, constatado nos trabalhos de Scholl (2001) e Warnock (1983). Todavia, neste trabalho o fator de amortecimento não foi determinado.

Outro ponto importante a ser considerado é que para a fonte padronizada a característica do material resiliente utilizado é o que influencia, pois se observa diferença no nível de pressão sonora entre os tipos de material resiliente utilizados. Já para a fonte não padronizada as combinações de piso com material resiliente têm resultados próximos aos da laje em "osso", mostrando, assim, que o que influencia mais são as características do piso utilizado. Entretanto, é fundamental o uso de material resiliente com os pisos, já que, quando os pisos são utilizados sozinhos, com o tipo de excitação que a fonte não padronizada causa, o nível de pressão sonora na câmara de recepção aumenta consideravelmente.

Por outro lado, ficou claro que o procedimento adicional do Anexo A da ISO 10140-4 (INTERNATIONAL..., 2010c) só proporciona diferença considerável (variação entre 4,7 e 7,4 dB) de nível de pressão sonora na frequência central de $80 \mathrm{~Hz}$; nas demais bandas o procedimento pode ser dispensado, uma vez que a diferença de nível de pressão sonora de ruído de impacto é pequena, variando na maioria dos casos em torno de $1 \mathrm{~dB}$. A questão que fica em aberto para ser discutida por novos estudos é se para frequências centrais mais baixas $(50$ e $63 \mathrm{~Hz})$ o procedimento adicional do Anexo A da ISO 10140-4:2010 também proporcionará considerável diferença como em $80 \mathrm{~Hz}$.

\section{Referências}

BRADLEY, J. S. Sound Insulation Issues. In: JOINT NSC-NRC WORKSHOP ON CONSTRUCTION TECHNOLOGIES, Taipei, 2004. Proceedings... Taipei, 2004.

CABEÇAS, A. J. S. Alargamento do Âmbito de Aplicação da Norma EN 12354-2 as Baixas Frequências. Lisboa, 2011.98 f. Dissertação (Mestrado em Engenharia Civil) - Instituto Superior Técnico, Universidade Técnica de Lisboa, Lisboa, 2011.

HOPKINS, C. Sound Insulation. London: Elsevier, 2007.

\section{INTERNATIONAL ORGANIZATION FOR} STANDARDIZATION. ISO 10140-1: a coustics: laboratory measurement of sound insulation of building elements: part 1: application rules for specific products. Geneva, 2010a.

\section{INTERNATIONAL ORGANIZATION FOR} STANDARDIZATION. ISO 10140-3: acoustics: laboratory measurement of sound insulation of building elements: part 3: measurement of impact sound insulation. Geneva, 2010b.

\section{INTERNATIONAL ORGANIZATION FOR} STANDARDIZATION. ISO 10140-4: acoustics: laboratory measurement of sound insulation of building elements: part 4: measurement procedures sound requirements. Geneva, 2010c.

\section{INTERNATIONAL ORGANIZATION FOR} STANDARDIZATION. ISO 10140-5: acoustics: laboratory measurement of sound insulation of building elements: part 5: requirements for test facilities and equipment. Geneva, 2010d. 
JEON, J. Y. et al. Evaluation of Floor Impact Sound Insulation in Reinforced Concrete Buildings. Acta Acustica United With Acustica, v. 90, p. 313-318, 2004.

JEON, J. Y. Subjective Evaluation of Floor Impact Noise Based on the Model of ACF/IACF. Journal of Sound and Vibration, v. 241, n. 1, 147-155, 2001.

JEON, J. Y.; SATO, S. Annoyance Caused by Heavyweight Floor Impact Sounds in Relation to the Autocorrelation Function and Sound Quality Metrics. Journal of Sound and Vibration, v. 311, p. 767-785, 2008.

KIM, K.-W. et al. Correlation Between Dynamic Stiffness of Resilient Materials and Heavyweight Impact Sound Reduction Level. Building and Environment, v. 44, n. 8, p. 1589-1600, 2009.

NEVES E SOUSA, A.; GIBBS, B. M. Low Frequency Impact Sound Transmission in Dwellings Through Homogeneous Concrete Floors and Floating Floors. Applied Acoustics, v. 72, n. 4, p. 177-189, 2011.

SCHOENWALD, S. et al. Approaches for Estimating Flanking Transmission for Heavy Impact Sources. In: InterNoise 2010, Lisboa, 2010. Proceedings... Lisboa, 2010.

SCHOENWALD, S.; ZEITLER, B.;

NIGHTINGALE, T. R. T. Influence of Receive Room Properties on Impact Sound Pressure Level Measured With Heavy Impact Sources. In: EUROREGIO 2010 CONGRESS ON SOUND AND VIBRATION, Ljubljana, 2010. Proceedings... Ljubljana, 2010.
SCHOLL, W. Impact Sound insulation: the standard tapping machine shall learn to walk! Building Acoustics, v. 8, n. 4, p. 245-256, 2001.

SHI, W.; JOHANSSON, C.; SUNDBÄCK, U. An Investigation of the Characteristics of Impact Sound Sources For Impact Sound Insulation Measurement. Applied Acoustics, v. 51, n. 1, p. 85-108, 1997.

WARNOCK, A. C. C. Floor Impact Noise and Foot Simulators. In: INTERNOISE, Edinburgh, 1983. Proceedings... Edingurgh, 1983.

WARNOCK, A. C. C. Floor Research at NRC Canada. In: CONFERENCE IN BUILDING ACOUSTICS, Dublin, 1998. Proceedings... Dublin, 1998.

WARNOCK, A. C. C. Low Frequency Impact Sound Transmission Through Floor Systems. In: INTERNOISE, Toronto, 1992. Proceedings... Toronto, 1992.

WARNOCK, A. C. C. Low-Frequency Impact Sound Transmission Through Floor Systems. In: InterNoise, Toronto, 2000. Proceedings... Toronto, 2000.

YOO, S. Y. et al. Measurement of Sound Field for Floor Impact Sounds Generated by Heavy/Soft Impact Sources. Acta Acustica United With Acustica, v. 96, p. 761-772, 2010.

\section{Agradecimentos}

Nossos agradecimentos à Coordenação de Aperfeiçoamento de Pessoal de Ensino Superior (Capes), pelo suporte financeiro mediante a concessão de bolsa de estudos.

Ana Maria Schuch Araújo

Curso de Arquitetura e Urbanismo | Universidade Federal da Fronteira Sul | ERS 135 - Km 72, $\mathrm{n}^{\circ} 200$ | Erechim - RS - Brasil | Caixa Postal 764 | CEP 99700-970 | Tel.: (54) 3321-7050 Ramal 7311 | E-mail: ana.araujo@uffs.edu.br

Stephan Paul

Departamento das Engenharias da Mobilidade | Universidade Federal de Santa Catarina | Rua Doutor João Colin, 2700, Santo Antônio | Joinville - SC - Brasil | CEP 89218-035 | Tel.: (48) 3721-7312 | E-mail: stephan.paul@ufsc.br

\section{Erasmo Felipe Vergara}

Departamento de Engenharia Mecânica, Centro Tecnológico | Universidade Federal de Santa Catarina | Campus Universitário, Trindade | Florianópolis - SC - Brasil | Caixa Postal 476 | CEP 88040-900 | Tel.: (48) 3721-9227 Ramal 228 | E-mail: e.f.vergara@ufsc.br

Revista Ambiente Construído

Associação Nacional de Tecnologia do Ambiente Construído

Av. Osvaldo Aranha, $99-3^{\circ}$ andar, Centro

Porto Alegre - RS - Brasil

CEP $90035-190$

Telefone: +55 (51) 3308-4084

Fax: +55 (51) 3308-4054

www.seer.ufrgs.br/ambienteconstruido

E-mail: ambienteconstruido@ufrgs.br

174 Araújo, A. M. S.; Paul. S.; Vergara, E. F. 